\title{
LIMITANTES DIAGNOSTICAS DE INFECCIONES OPORTUNISTAS EN PACIENTES CON VIH.
}

\section{Limited diagnosis of opportunistic infections in Hiv-patients.}

*Karen Lujan, ${ }^{* * K a r e n ~ E r a z o . ~}$

\section{INTRODUCCIÓN}

Las enfermedades oportunistas son aquellas que se presentan generalmente como consecuencia de la severa inmunodepresión en pacientes con infección por el virus de inmunodeficiencia humana (VIH). El nivel de inmunodepresión especialmente con linfocitos CD4 $<200$ células/ml, es el principal factor de riesgo. ${ }^{(1)}$

El Hospital Nacional Mario Catarino Rivas es el centro con la cohorte más grande de pacientes con VIH recibiendo atención médica, el clínico día a día se enfrenta no solo al manejo de la infección por VIH en sí, sino también al manejo de otros aspectos de la misma; uno de los más frecuentes son las infecciones oportunistas (IO).

Las más frecuentes, Tuberculosis en todas sus manifestaciones, Histoplasmosis, Toxoplasmosis, Candidiasis e infección por citomegalovirus. Durante el año 2016 se presentaron un total de 211 nuevos casos de infecciones oportunistas en el servicio de atención integral (SAI) del Hospital Nacional Dr. Mario Catarino Rivas (HNMCR). De esta cohorte, el $69 \%$ correspondió al sexo masculino siendo el rango de edad más afectado 21 - 40 años. Con el presente artículo se pretende dar a conocer las limitantes en el diagnóstico de las diferentes infecciones oportunistas encontradas en pacientes con VIH durante el año 2016. (Ver Tabla No. 1).

*Médico General del Servicio de Atención Integral, Hospital Nacional Mario Catarino Rivas. (SAI/HNMCR)

${ }^{* *}$ Médico Pediatra, Máster en VIH, Coordinadora del SAI/HNMCR. Profesor Titular de la Escuela Universitaria de las Ciencias de la Salud, Universidad Nacional Autónoma de Honduras en el Valle de Sula. EUCS, UNAH-VS

Dirigir correspondencia a: karenerazo@yahoo.com

Recibido: 16 de mayo 2017

Aprobado: 19 de septiembre 2017
Tabla No. 1: Principales infecciones oportunistas durante el año 2016 en el SAl/HNMCR.

\begin{tabular}{|r|llll|}
\hline $\begin{array}{r}\text { Infecciones } \\
\text { oportunistas }\end{array}$ & \multicolumn{2}{c}{$\begin{array}{c}\text { Número de } \\
\text { Casos }\end{array}$} & Hombres & Mujeres \\
\hline $\begin{array}{r}\text { tuberculosis } \\
\text { toxoplasmosis }\end{array}$ & $(56)$ & $31 \%$ & $(46) 69.6 \%$ & $(20) 30.4 \%$ \\
candidiasis & $(60)$ & $25 \%$ & $(34) 65.3 \%$ & $(18) 34.7 \%$ \\
citomegalovirus & $(7)$ & $3.3 \%$ & $(6) 85.7 \%$ & $(1) 14.3 \%$ \\
histoplasmosis & $(10)$ & $4.7 \%$ & $(8) 80 \%$ & $(2) 20 \%$ \\
criptococosis & $(16)$ & $7.5 \%$ & $(12) 75 \%$ & $(4) 25 \%$ \\
Total & $(211)$ & $100 \%$ & $(146) 69 \%$ & $(65) 31 \%$ \\
\hline
\end{tabular}

Fuente: Base de datos del Servicio de Atención Integral HNMCR

\section{TUBERCULOSIS (TB)}

La tuberculosis es una de las 10 principales causas de mortalidad en el mundo. En 2015, 10,4 millones de personas enfermaron de tuberculosis y 1,8 millones murieron por esta enfermedad (entre ellos, 0,4 millones de personas con $\mathrm{VIH})$. Más del $95 \%$ de las muertes por tuberculosis se producen en países de ingresos bajos y medianos. ${ }^{(2,3)}$

En pacientes con tuberculosis, el tracto respiratorio es uno de los órganos que más frecuentemente se ve afectado en los pacientes con infección por $\mathrm{VIH}$. Hay otros órganos que se pueden ver afectados en la tuberculosis extra pulmonar entre ellos los ganglios linfáticos suele ser los de mayor incidencia, en el SAI del HNMCR, esa fue la tuberculosis extra pulmonar más frecuente seguido de la tuberculosis diseminada, tuberculosis meníngea y en último lugar la tuberculosis cutánea.

Esta infección oportunista es más frecuente en pacientes del sexo masculino con estado de inmunosupresión característico menor de 200 celulas $/ \mathrm{mm}^{3} \mathrm{CD} 4$, sobre todo en paciente en los cuales no han tenido terapia antirretroviral y como reactivación de tuberculosis en aquellos casos de pacientes que no cum- 
plieron tratamiento profiláctico con isoniacida y que tienen compromiso del estado inmunológico por falla virológica o falla clínica; y en aquellos que están en abandono de terapia retroviral. Sin embargo puede presentarse en pacientes sin inmunosupresión. ${ }^{(2)}$

La co-infección tuberculosis/VIH es uno de los problemas más importantes que afronta la salud pública y se da con mayor frecuencia en países del tercer mundo. Se calculan más de 14 millones de personas viviendo con la co-infección; el 30\% de las muertes de enfermedad avanzada por VIH o Sida están relacionadas con Tuberculosis (TB) y el $6 \%$ de las muertes de pacientes con TB están relacionadas con $\mathrm{VIH} .{ }^{(3)}$

En el HNMCR durante el año 2016 la IO más común fue la TB Pulmonar en un $31 \%$ siendo más frecuente en varones. Las dificultades en obtener un diagnóstico temprano fueron: la falta de sospecha clínica, el tamizaje para TB escaso y en pacientes en los cuales presentaron rayos $\mathrm{X}$ altamente subjetivas con la sospecha clínica pero no brindaron muestras adecuadas de esputo. El inicio del tratamiento se retrasó por la negativa de los Centros de Salud de dar terapia antifímica por tener un seriado de BAAR negativa, por lo que se hace necesario concientizar al personal de salud en este aspecto.

En los casos de TB extra pulmonar específicamente en TB ganglionar la limitante principal son las estadías hospitalarias prolongadas en espera de toma de biopsia por el servicio de cirugía y la demora del resultado de esta por el servicio de patología.

Se cuenta con el acceso al adenosina desaminasa (ADA) sin embargo hay lentitud en la entrega de resultados, y el cultivo como todos sabemos es un método diagnóstico a largo plazo.

No podemos negar que la llegada del gene xpert para diagnóstico rápido y detección de resistencia a rifampicina ha sido de enorme utilidad sobre todo por lo rápido de los resultados permitiendo hacer diagnósticos más oportunos.

\section{TOXOPLASMOSIS CEREBRAL}

Se señala que alrededor de un tercio de los pacientes con infecciones por el VIH presentan complicaciones en el sistema nervioso central, la más frecuente la toxoplasmosis cerebral que provocan considerable morbilidad y mortalidad y que las principales manifestaciones neurológicas pueden ser focales o generalizados, siendo la cefalea, la confusión, la fiebre y convulsiones los más importantes. Clínicamente predomina un síndrome compatible con lesiones ocupantes; son comunes además la presencia de hemiparesias, deficiencias visuales, hipertensión endocraneana, elementos neurológicos focales, compromiso de pares craneanos, trastornos de conciencia, alteraciones siquiátricas y somnolencia. Raramente se encuentra rigidez de nuca. La sintomatología es variable dependiendo de la localización de las lesiones y su número. ${ }^{(4)}$

La toxoplasmosis afectó a $25 \%$ de la población con $\mathrm{IO}$ en el año 2016 en el HNMCR, siendo más frecuente en los hombres con 34 casos el rango de edad más afectado fueron los individuos entre 21 a 40 años. (Ver Tabla 1). Las dificultades diagnósticas enfrentadas fueron la escases de reactivos para las pruebas serológicas, la ausencia de materiales para toma de muestras de líquido cefalorraquídeo, escases de estudio de imágenes como ser TAC cerebral. En cuanto al tratamiento se tuvo acceso al mismo todo el año, sin embargo en el mes de diciembre hubo escases de pirimetamina el cual es el fármaco que actúa sinérgicamente con las sulfonamidas para destruir los taquizoitos del toxoplasma.

\section{CRIPTOCOCOSIS}

La criptococosis es una enfermedad micótica oportunista, grave, causada por un hongo levaduriforme y encapsulado denominado Cryptococcus neoformans. Sus dos variedades; Cryptococcus neoformans variedad neoformans (serotipos A y D) y Cryptococcus neoformans variedad gattii (serotipos $B$ y C) son responsables de la enfermedad en el hombre. La meningitis criptococócica afecta a pacientes inmuno-comprometidos. 
De los pacientes con enfermedad avanzada por VIH, alrededor del 5 al $10 \%$ se infectan con Cryptococcus, de éstos el $90 \%$ desarroIlan meningitis por Cryptococcus particularmente cuando el recuento de CD4 es menor a 100 células $/ \mathrm{mm} 3 .{ }^{(5)}$ Es más frecuente en varones que en mujeres con una relación de $4: 1$, tal vez debido a la mayor exposición de los hombres a este microorganismo. Este hongo genera la infección oportunista de inicio en más de $50 \%$ de todos los enfermos con enfermedad avanzada por VIH; presenta una tasa de mortalidad del 6 al 25\% durante las dos primeras semanas de tratamiento y del 40 al $70 \%$ a los 12 meses. La forma clínica de presentación generalmente tiene una evolución subaguda o crónicas se caracteriza por cefalea, fiebre y malestar general, posteriormente puede observarse síndrome de hipertensión endocraneana, edema de papila, diplopía, nauseas, vómitos $y$ al evolucionar la enfermedad el paciente presenta rigidez de nuca, hipersensibilidad del cuello. En individuos inmunocompetentes la infección es autolimitada y poco sintomática. ${ }^{(5)}$

En los pacientes del Servicio de Atención Integral (SAI) con IO en el 2016, el $7.5 \%$ presentó criptococosis meníngea (Ver Tabla No. 1). Las dificultades diagnósticas presentadas durante el año 2016, ausencia de antígeno para criptococosis, falta de reactivos laboratoriales en los estudios de líquido cefalorraquídeo, el acceso intermitente a TAC cerebral, la ausencia de pruebas serológicas. En cuanto al acceso al tratamiento durante todo el año hubo disponibilidad de anfotericina y fluconazol.

\section{HISTOPLASMOSIS}

La histoplasmosis es la infección causada por el hongo Histoplasma capsulatum ha sido reportada en todos los continentes. El hongo se desarrolla en el suelo con excretas de aves y murciélagos, donde es capaz de producir abundantes microconidios, que al ser inhalados por el hombre son capaces de causar la infección. El cuadro clínico puede variar, desde infecciones asintomáticas hasta cuadros diseminados graves que involucran a uno o varios órganos y sistemas y que afectan sobre todo a pacientes con VIH, neoplasias hematológicas, con trasplantes u otras inmunodeficiencias. En individuos con $\mathrm{VIH}$ suele aparecer en estadios avanzados de la infección, asociada con recuentos inferiores a 100 linfocitos CD4/ $\mathrm{mm}^{3}$.

El diagnóstico suele ser difícil, pues sus características clínicas y resultados de exámenes de laboratorio de rutina son comunes a otras complicaciones asociadas a la enfermedad avanzada por $\mathrm{VIH}$, como tuberculosis, neumocistosis y demás infecciones sistémicas. Además, la demostración del hongo requiere un laboratorio de microbiología con experiencia en identificar la levadura, sus antígenos o anticuerpos específicos.

El pronóstico es bueno en los pacientes con enfermedad leve, y en quienes el diagnóstico rápido permite la administración temprana del tratamiento. Sin embargo, la tasa de mortalidad puede ser alta, especialmente en pacientes inmunosuprimidos o con histoplasmosis grave (presencia de hipotensión que requiere vasopresores, necesidad de ventilación mecánica y deterioro del estado de conciencia), en quienes la tasa de mortalidad puede ascender a $47-70 \% .{ }^{(6)}$

La Histoplasmosis se presentó en un $4.7 \%$ de la población descrita durante el año 2016 (Ver Tabla 1). Los problemas diagnósticos se encontraron debido a que la observación de la levadura en fluidos y tejidos orgánicos, requiere mucha experiencia por el personal de laboratorio y no se cuenta con suficiente personal capacitado para su identificación, además, no se cuenta con los medios para realizar pruebas de detección de anticuerpos y antígenos específicos ni reacción en cadena de polimerasa, muchas veces el único medio diagnóstico disponible ha sido el buffy coat que es un extendido rico en células blancas del centrifugado capilar de sangre periférica teñido con tinción de gram. 
En cuanto al acceso del tratamiento se ha contado con anfotericina, sin embargo no se tiene a disposición itraconazol.

\section{CANDIDIASIS}

La candidiasis mucocutánea es la presentación más común, en los pacientes VIH positivos con recuentos de CD4 inferiores a 300 células/mm3; La candidiasis oro faríngea se presenta como placas blanquecinas que se pueden remover con el baja lenguas y se localizan principalmente en el dorso de la lengua, el paladar blando, las amígdalas y la mucosa oral. También pueden encontrarse placas eritematosas sin el característico exudado. Puede extenderse a hipofaringe y comprometer el esófago. La candidiasis esofágica es una de las entidades definitorias de sida y una de las infecciones oportunistas más ampliamente reportadas en la literatura. ${ }^{(6)}$ Desde las descripciones iniciales del sida, las descripciones oportunistas de la cavidad bucal han demostrado un lugar preponderante y han tenido un importante valor diagnóstico y pronostico, ya que $90 \%$ de sujetos con VIH presentan lesiones bucales, con un enorme valor clínico, pudiendo ser la primera manifestación en los pacientes VIH y/o SIDA, constituyéndose no solo como indicador precoz de la enfermedad si no como marcador de severidad y marcador pronóstico de la misma. ${ }^{(7)}$

En el HNMCR la candidiasis orofaríngea fue la infección oportunista por hongos más frecuente en un $28.5 \%$, siendo la población masculina más afectada en un $66.6 \%$ entre las edades de 21 a 40 años (Ver Tabla 1). El diagnóstico fue fundamentalmente clínico, sin embargo, en los casos de afectación esofágica era necesario la endoscopía, teniendo en algunas ocasiones retrasos en las programaciones de dicho procedimiento.

\section{CITOMEGALOVIRUS}

El citomegalovirus humano $(\mathrm{CMVH})$ es un patógeno oportunista que pertenece a la familia beta herpes virus. CMVH, posee un genoma de ácido desoxirribonucleico (ADN) de doble cadena, con aproximadamente 230 kpb y 200 marcos de lectura abiertos. La infección por $\mathrm{CMVH}$ se manifiesta como retinitis, esofagitis, colitis o encefalitis y representa la principal complicación y primera causa de morbi-mortalidad en pacientes inmunocomprometidos. ${ }^{(8)}$ Del 20 al $40 \%$ de los individuos infectados con el virus de la inmunodeficiencia humana que no están bajo tratamiento antirretroviral, desarrollan enfermedad a causa de CMVH. Se ha observado que la coinfección de $\mathrm{VIH}$ y CMVH en individuos que presentan una cuenta de linfocitos CD4+ menor a $50 / \mu \mathrm{L}$, incrementa el riesgo de presentar severas complicaciones. ${ }^{(9,10)}$

En el SAl se presentaron 7 casos de infección por citomegalovirus correspondiendo al $3.3 \%$ de las infecciones oportunistas, predominando en hombres en un $85.7 \%$. (Ver Tabla No. 1). La dificultad en la realización del diagnóstico radica en no poder contar con la reacción en cadena de polimerasa, lo que limita a realizar pruebas de anticuerpos, y a la detección de cuerpos de inclusión en orina. En cuanto al tratamiento no se ha tenido acceso a valganciclovir de manera continua.

En conclusión Aunque el acceso a la terapia antirretroviral de gran actividad es mayor actualmente, aun el clínico debe prepararse para continuar diagnosticando y tratando las infecciones oportunistas en el paciente con $\mathrm{VIH}$, pero indudablemente necesita tener todo el recurso a la mano para poder realizar diagnósticos precoces y oportunos pues de esto depende el pronóstico de estos pacientes. 


\section{REFERENCIAS BIBLIOGRÁFICAS}

1. Universidad de la República de Uruguay. Manual de práctica clínica: infecciones oportunistas en VIH. [Internet] 2016 [Actualizada 2017, Citado 23 de abril de 2017]. Disponible en: http://www.gastro. hc.edu.uy/gastro/dt_portfolios/manual -de-practica-clinica-infecciones-oportu nistas-en-hiv/.

2. Peñuela-Espalsa $M$, Vásquez Beltrán $M$, De La Rosa Barraza K, Hernández Tapia H, Collazos Daza J, Yanes Miranda Y. Factores asociados a la coinfección $\mathrm{VIH} / \mathrm{SIDA}$ - tuberculosis Barranquilla (Colombia), 2003-2004. Rev Sal Unin. [Internet]. 2006 [Citado 1 de mayo de 2017]; 22(1). Disponible en: http:// sear ch.proquest.com/openview/70dcfb 71 b0d82e43800f309784d53104/1?pq-or igsite=gscholar \&cbl=2027436 .

3. Palou E. Tuberculosis y sida: una co-infeccion eficiente. Rev Méd hondur [Internet]. 2010 [citado 24 de abril de 2017]; 78(1). Disponible en: http://www.bvs.hn/ $\mathrm{RMH} / \mathrm{pdf} / 2010 / \mathrm{pdf} / \mathrm{Vol} 78-1-2010-11$. pdf.

4. Hernández I, García S. Toxoplasmosis: infección oportunista en pacientes con el síndrome de inmunodeficiencia adquirida. Rev Biomed [Internet]. 2003 [Citado 24 de abril de 2017]; 14(2):101-111. Disponible en: http://www.revbiomed.ua dy.mx/pdf/rb031427.pdf.

5. Castro M, Córdova A. Características clínicas y laboratoriales de la coinfeccion $\mathrm{VIH}-\mathrm{SIDA}$ y criptococosis meningea en el Hospital Clínico Viedma de Cochabamba, Bolivia. Gac Médica Boliv. 2014; 37(2):64-7.
6. Gonzalez A, Tobón A. Infecciones-micoticas-oportunistas-en-pacientescon-VIH-SIDA. Asociación Colombiana de Infecto logia [Internet] 2006. [citado 24 de abril de 2017]; 10(4): 279-288. Disponible en: file:///C:/Users/Admin/ Downloads/Infecciones_micoticas_opor tunistas_en_pacientes_co.pdf.

7. Tovar V. Albornoz E, Guerra M, Lazarde J Prevalencia de candidiasis bucal en pacientes vih/sida: estudio retrospectivo. acta odontol venez. [internet]. 2004 [Citado 24 de abril de 2017]; 42(2). Disponible en: http://www.actaodontologica. com/ediciones/2004/2/prevalencia_can didiasis_bucal_pacientes_vih_sida.asp.

8. Suárez-Pichilingue G, Verona-Rubio R. Coinfección pulmonar por citomegalovirus y Cryptoccocus sp. en una paciente con infección por VIH. Rev Soc Peru Med Interna. 2010;23(1):24-7.

9. González Calixto E, Ruiz Tachiquin ME, Burgueño Ferreira J, Aguilera P, Espinoza Rojo AM. Detección temprana y altamente sensible de citomegalovirus en muestras de plasma humano VIH-positivas. Bioquimica. [Internet] 2009 [citado 24 de abril de 2017]; 34(3): 129-136. Disponible en: http://www.medigraphic. $\mathrm{com} / \mathrm{pdfs} / \mathrm{bioquimia/bq-2009/bq093d}$. pdf.

10. Centro Nacional de Excelencia Tecnológica en Salud (CENETEC). Guías prácticas clínicas: prevención diagnóstico y tratamiento de la infección de la citomegalovirus en la edad pediátrica. [Internet] Guauhtemoc: CENETEC; 2013. [citado 2 de mayo de 2017]. Disponible en:http:// www.cenetec.salud.gob.mx/descargas/ gpc/CatalogoMaestro/IMSS_610_13_Cl TOMEGALOVIRUS/610GER.pdf. 